\title{
Hypovirulence-Associated Double-Stranded RNA from Sclerotinia homoeocarpa Is Conspecific with Ophiostoma novo-ulmi Mitovirus 3a-Ld
}

\author{
F. Deng, R. Xu, and G. J. Boland
}

First and third authors: Department of Environmental Biology, and second author: Laboratory Service Division, University of Guelph, Guelph, ON, N1G 2W1, Canada.

Accepted for publication 6 June 2003.

\begin{abstract}
Deng, F., Xu, R., and Boland, G. J. 2003. Hypovirulence-associated double-stranded RNA from Sclerotinia homoeocarpa is conspecific with Ophiostoma novo-ulmi mitovirus 3a-Ld. Phytopathology 93:14071414.

The nucleotide sequence of the hypovirulence-associated doublestranded RNA (dsRNA) in hypovirulent isolate Sh12B of Sclerotinia homoeocarpa, the causal agent of dollar spot of turf grass, was determined. This large dsRNA (L-dsRNA) is 2,632 bp long and is A and U rich (61.0\% $\mathrm{A}+\mathrm{U}$ residues). One strand of this dsRNA contains an open reading frame $(\mathrm{ORF})$ with the potential to encode a protein of 720 amino acids. This ORF contains 12 UGA codons, predicted to encode tryptophan in ascomycete mitochondria, and has a codon bias typical of mito-

chondrial genes, which is consistent with a mitochondrial localization of this dsRNA. The amino acid sequence contains conserved motifs typical of RNA-dependent RNA polymerases (RdRps). Sequence analyses of the nucleotide and RdRp-like protein revealed that the L-dsRNA is homologous with previously characterized mitochondrial viruses and dsRNAs from other phytopathogenic fungi, and shares $92.4 \%$ nucleotide and 95.1\% amino acid sequence identities with the Ophiostoma novo-ulmi mitovirus 3a-Ld from Ophiostoma novo-ulmi, the causal agent of Dutch elm disease. The results indicate that these two dsRNAs are conspecific. This is the first report that a hypovirulence-associated dsRNA virus naturally occurs in two taxonomically distinct fungi, and indicates that horizontal transmission of this dsRNA virus may have occurred between these fungi.
\end{abstract}

The presence of double-stranded RNA (dsRNA) viruses has been reported in all classes of fungi; however, in most cases, these infections have not been associated with apparent disease symptoms or other phenotypes. In some phytopathogenic fungi, however, dsRNA viruses are associated with reduced virulence (hypovirulence) and other phenotypes such as reduced growth, sporulation, or pigmentation (32). These hypovirulence-associated dsRNA viruses can be transmitted from infected fungal isolates to vegetatively compatible isolates during hyphal anastomosis. Transmission results in the conversion of the recipient isolate to hypovirulence and provides the basis for a unique mechanism of biological control $(2,30)$. Hypovirulence-associated dsRNAs have been extensively studied in some phytopathogenic fungi, such as Cryphonectria parasitica (Murrill) Barr, Rhizoctonia solani Kühn, and Ophiostoma novo-ulmi Brasier. Several hypovirulence-associated, unencapsidated dsRNAs have been characterized and classified into the two new virus families, Hypoviridae (Hypovirus) (19) and Narnaviridae (Mitovirus) (48). In addition, molecular analyses of these dsRNAs have provided evidence as to how these agents may cause hypovirulence-associated phenotypes $(12,27$, $31,41)$.

Dollar spot, caused by Sclerotinia homoeocarpa F. T. Bennett, is a widespread and persistent disease of many turf grass species (46). Hypovirulent isolates of $S$. homoeocarpa have been reported and the hypovirulent phenotypes were associated with up to two dsRNAs (49), but only the large (L)-dsRNA (2.6 kb) was shown to be associated with hypovirulence. The potential of utilizing hypovirulent isolates of $S$. homoeocarpa as biological control agents

Corresponding author: G. J. Boland; E-mail address: gboland@uoguelph.ca

Publication no. P-2003-0910-01R

(C) 2003 The American Phytopathological Society was evaluated in both controlled environment and field conditions. In growth-room conditions, hypovirulent isolate Sh12B suppressed disease by 51 to $90 \%$. In artificially infested field plots, the hypovirulent isolate suppressed disease by $80 \%$ and disease suppression was significant for 1 year postinoculation. In naturally infested field plots, the hypovirulent isolates suppressed disease by up to $58 \%$ and, in most plots, disease suppression was equivalent to treatment with the fungicide chlorothalonil (50).

A major objective of studies on hypovirulence-associated dsRNA in plant pathogenic fungi is to unravel the mechanisms by which these dsRNAs or their specific gene products may perturb virulence expression in their fungal hosts and, ultimately, to utilize such knowledge in developing biological control measures for management of plant diseases. This requires detailed knowledge of the structural and functional properties of hypovirulenceassociated dsRNAs. Therefore, we have cloned and sequenced the hypovirulence-associated dsRNA in $S$. homoeocarpa and, in this article, we report that the hypovirulence-associated dsRNA (LdsRNA) in S. homoeocarpa is conspecific with O. novo-ulmi mitovirus 3a-Ld from O. novo-ulmi, the causal agent of Dutch elm disease. This is the first report that a hypovirulence-associated dsRNA virus naturally occurs in two taxonomically distinct fungi.

\section{MATERIALS AND METHODS}

Isolates. Hypovirulent isolate Sh12B of S. homoeocarpa has been described by Zhou and Boland (49,50). This isolate was maintained on potato dextrose agar (PDA; Difco Laboratories, Detroit) at $4{ }^{\circ} \mathrm{C}$ and cultured at room temperature $\left(20\right.$ to $\left.22^{\circ} \mathrm{C}\right)$ for RNA isolation. The diseased isolate $\log 1 / 38 \mathrm{~d}^{2}$ of $O$. novo-ulmi was provided by C. M. Brasier (Forest Research Station, Alice Holt Lodge, Farnham, Surry, UK). This isolate was grown and stored on malt extract agar (MEA; Difco Laboratories) medium. 
RNA isolation. Colonies of hypovirulent isolate Sh12B of $S$. homoeocarpa and diseased isolate $\log 1 / 38 \mathrm{~d}^{2}$ of $O$. novo-ulmi were grown on cellophane membrane on PDA and MEA, respectively. After 5 to 14 days of growth, mycelium was scraped from the membrane, frozen with liquid nitrogen, and ground to a fine powder. dsRNAs were extracted from the powder using DNAzol (Molecular Research Center, Inc., Cincinnati, OH) according to the manufacturer's instructions. For separation of single-stranded RNA (ssRNA) and dsRNA, total nucleic acids were extracted using RNA extraction buffer $(200 \mathrm{mM}$ Tris base, $400 \mathrm{mM} \mathrm{KCl}$, $200 \mathrm{mM}$ sucrose, $35 \mathrm{mM} \mathrm{MgCl} 2,25 \mathrm{mM}$ EDTA, pH 9.0). After phenol and chloroform extraction, ssRNA and dsRNA were separated by precipitating ssRNA with $2 \mathrm{M} \mathrm{LiCl} \mathrm{(3)} \mathrm{and} \mathrm{dsRNA} \mathrm{with}$ $5 \mathrm{M} \mathrm{LiCl}$ (14) as described by Hong et al. (21).

cDNA synthesis. The target dsRNA was gel purified and double-stranded (ds)cDNA synthesis was conducted using Universal RiboClone cDNA Synthesis System (Promega Corp., Madison, WI) according to the manufacturer's instructions. Briefly, firststrand cDNA was synthesized in $15 \mu \mathrm{l}$ of reaction mixture containing $10 \mu \mathrm{l}$ of purified dsRNA, $2 \mu \mathrm{l}$ of random hexameric primer $(0.5 \mu \mathrm{g} / \mu \mathrm{l})$, and $3 \mu \mathrm{l}$ of nuclease-free water. The reaction was incubated at $70^{\circ} \mathrm{C}$ for $10 \mathrm{~min}$, and cooled on ice for $10 \mathrm{~min}$. Then, $5 \mu \mathrm{l}$ of first strand buffer $(5 \times), 1 \mu \mathrm{l}$ of RNasin ribonuclease inhibitor (40 U), $2.5 \mu$ of sodium pyrophosphate $(40 \mathrm{mM}), 1.5 \mu \mathrm{l}$ of AMV reverse transcriptase $(30 \mathrm{U})$, and $0.5 \mu \mathrm{l}$ of nuclease-free water were added. The reaction was incubated at $37^{\circ} \mathrm{C}$ for $60 \mathrm{~min}$ and cooled on ice for $5 \mathrm{~min}$. Second-strand cDNA was synthesized in $50 \mu \mathrm{l}$ of reaction volume, which was composed of $10 \mu \mathrm{l}$ of first-strand reaction solution, $20 \mu \mathrm{l}$ of second-strand buffer $(2.5 \times)$, $1.1 \mu \mathrm{l}$ of DNA polymerase I (11.5 U), $0.2 \mu \mathrm{l}$ of RNase $\mathrm{H}(0.4 \mathrm{U})$, and $18.7 \mu \mathrm{l}$ of nuclease-free water. The reaction was incubated at $14^{\circ} \mathrm{C}$ for $3 \mathrm{~h}$, followed by $70^{\circ} \mathrm{C}$ for $10 \mathrm{~min}$. The synthesized dscDNA was purified using QIAamp spin column (Qiagen, Chatsworth, CA), ligated to the vector pBluscript II (Stratagene, La Jolla, CA), and transformed into Escherichia coli DH5 $\alpha$. cDNA clones were screened by Northern blotting using randomly primed cDNA probes prepared from the dsRNA.

Primer walking and sequencing. After sequencing a cDNA clone, the full-length sequence of the L-dsRNA then was completed by primer walking and rapid amplification of cDNA ends (RACE) clones. In primer walking, the synthesized dscDNAs were ligated to an EcoRI Adaptor and polymerase chain reaction (PCR) was performed to amplify the cDNA fragment using HotStarTaq DNA polymerase (Qiagen). The primers used for amplification of the cDNA fragment were EcoRI Adaptor primer (5'AATTCCGTTGACTGCTGTCG-3') and specific primers corresponding to the known sequence of the dsRNA. The PCR products were separated by $7 \%$ polyacrylamide gel electrophoresis, and the target DNA band was re-amplified. The re-amplified PCR products were directly sequenced and new specific primers were designed using GeneWorker 2.5 software based on the sequences obtained. This PCR-sequencing procedure was conducted to provide overlapping sequences of the dsRNA and both strands of the dsRNA were sequenced.

Complementary DNA clones of the ends of the L-dsRNA were obtained by the RACE procedure (17) using a 5'/3' RACE kit (Roche, Indianapolis, IN). Briefly, the dsRNA was denatured at $70^{\circ} \mathrm{C}$ for $10 \mathrm{~min}$ and cDNA was synthesized using reverse transcriptase. The purified cDNA was tailed with A residues using terminal transferase and the poly (A) tailed cDNA was amplified using the primer 5'-GACCACGCGTATCGATGTCGAC(T) ${ }_{16} \mathrm{~V}-3^{\prime}$ and a nested primer that is specific to the known sequence of the dsRNA. The 5' RACE PCR products were cloned into the vector pGEM-T Easy (Promega Corp.) and transformed into E. coli $\mathrm{DH} 5 \alpha$. Sequences of the cDNA clones and PCR products were obtained by the Sanger chain termination method (37) using an ABI PRISM sequencer, and the entire L-dsRNA molecule was sequenced from overlapping clones on both strands. In addition, the sequence of the L-dsRNA was verified by sequencing reverse transcription (RT)-PCR products. Sequence analysis was per-
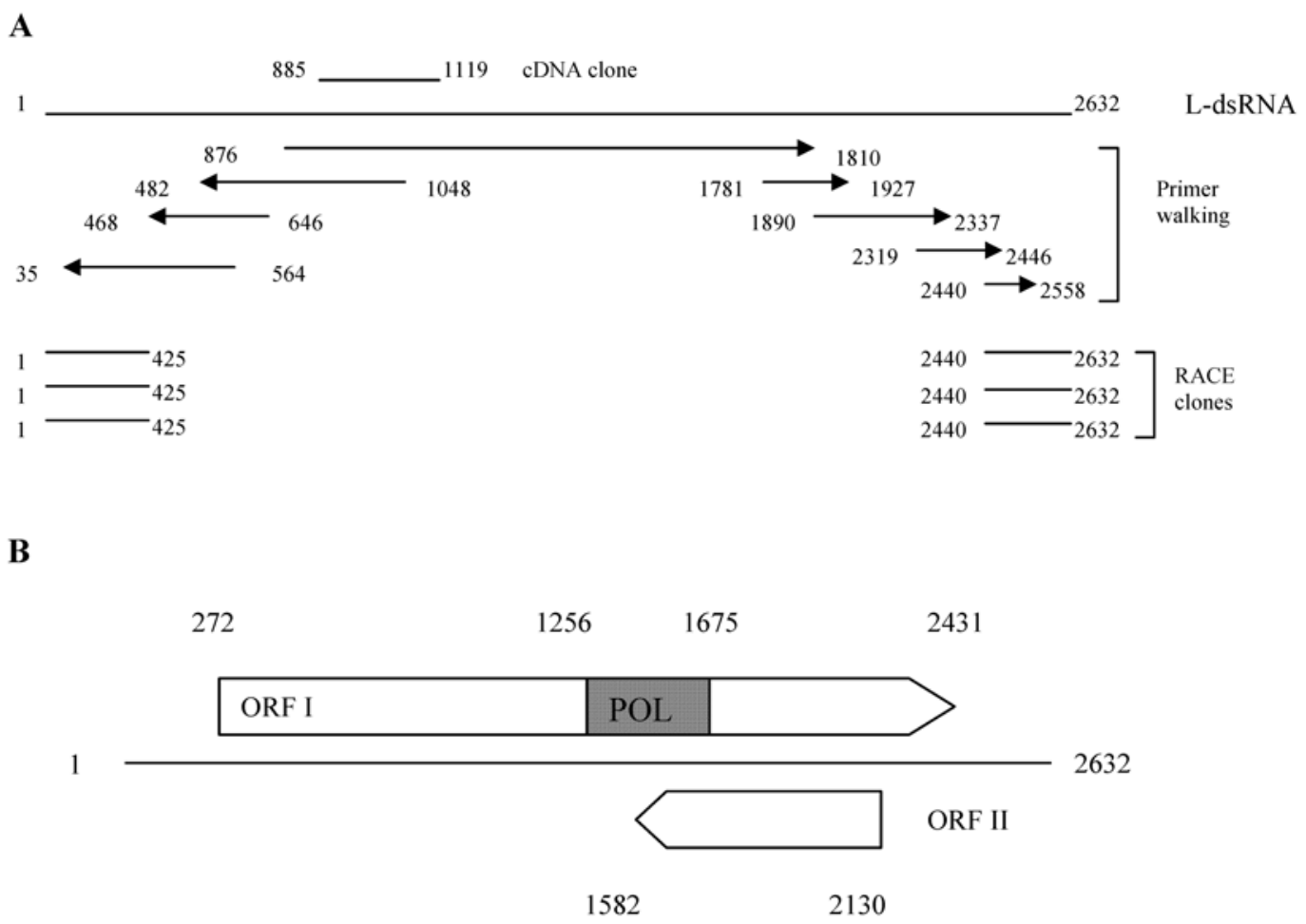

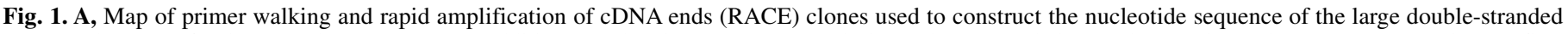

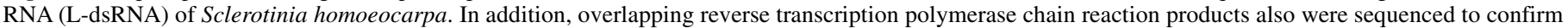

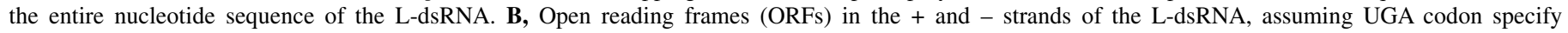

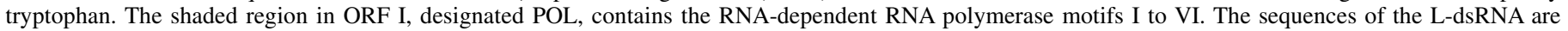
available from the GenBank database with accession number AY172454. 
formed using the Needle program of the European Molecular Biology Open Software Suite (EMBOSS), and multiple sequence alignments were done with CLUSTAL W (42). The cDNA clones and PCR products (primer walking) used to obtain the complete sequence of the L-dsRNA are illustrated in Figure 1A.

Northern hybridization. dsRNA and ssRNA fractions were separated by electrophoresis in a $1.4 \%$ agarose gel. After electrophoresis, the gels were soaked in $50 \mathrm{mM} \mathrm{NaOH}$ and $150 \mathrm{mM}$ $\mathrm{NaCl}$ for $15 \mathrm{~min}$, then in $10 \times \mathrm{SSC}(1 \times \mathrm{SSC}$ is $0.15 \mathrm{M} \mathrm{NaCl}$ plus
$0.015 \mathrm{M}$ sodium citrate) for $10 \mathrm{~min}$, before being transferred to a nylon membrane. Prehybridization was conducted in prehybridization buffer (5x SSC, $0.1 \%$ sodium-lauroylsarcosine, $0.02 \%$ sodium dodecyl sulfate [SDS], and $1 \%$ blocking reagent) for 4 to $6 \mathrm{~h}$ at $42^{\circ} \mathrm{C}$ according to the manufacturer's instructions (Boehringer Mannheim, Germany). Digoxigenin-labeled strand-specific DNA probes were prepared using the PCR procedure described by Finckh et al. (16). The primers used to generate ssDNA probes were $2036 \mathrm{~F}$ (5'-CTGAACCAGCTGGCTTTGAAGTC-3') and
A

L-dsRNA
OnuMV3a-Ld
RsM2-1A1
CpMV1-NB631
OnuMV6-Ld
OnuMV4-Ld
OnuMV5-Ld

L-dsRNA

OnUMV3a-Ld

RsM2 - 1A1

CpMV1-NB631

OnuMV6-Ld

OnuMV4 - Ld

OnuMV5-Ld

L-dsRNA OnUMV3a-Ld

RsM2 - 1A1

CpMV1-NB631

OnUMV 6-Ld

OnuMV4 - Ld

OnuMV5-Ld

L-dsRNA

OnuMV3a

RsM2 - 1

CpMV1-NB631

OnuMV6-Ld

OnuMV4-Ld

OnuMV5-Ld
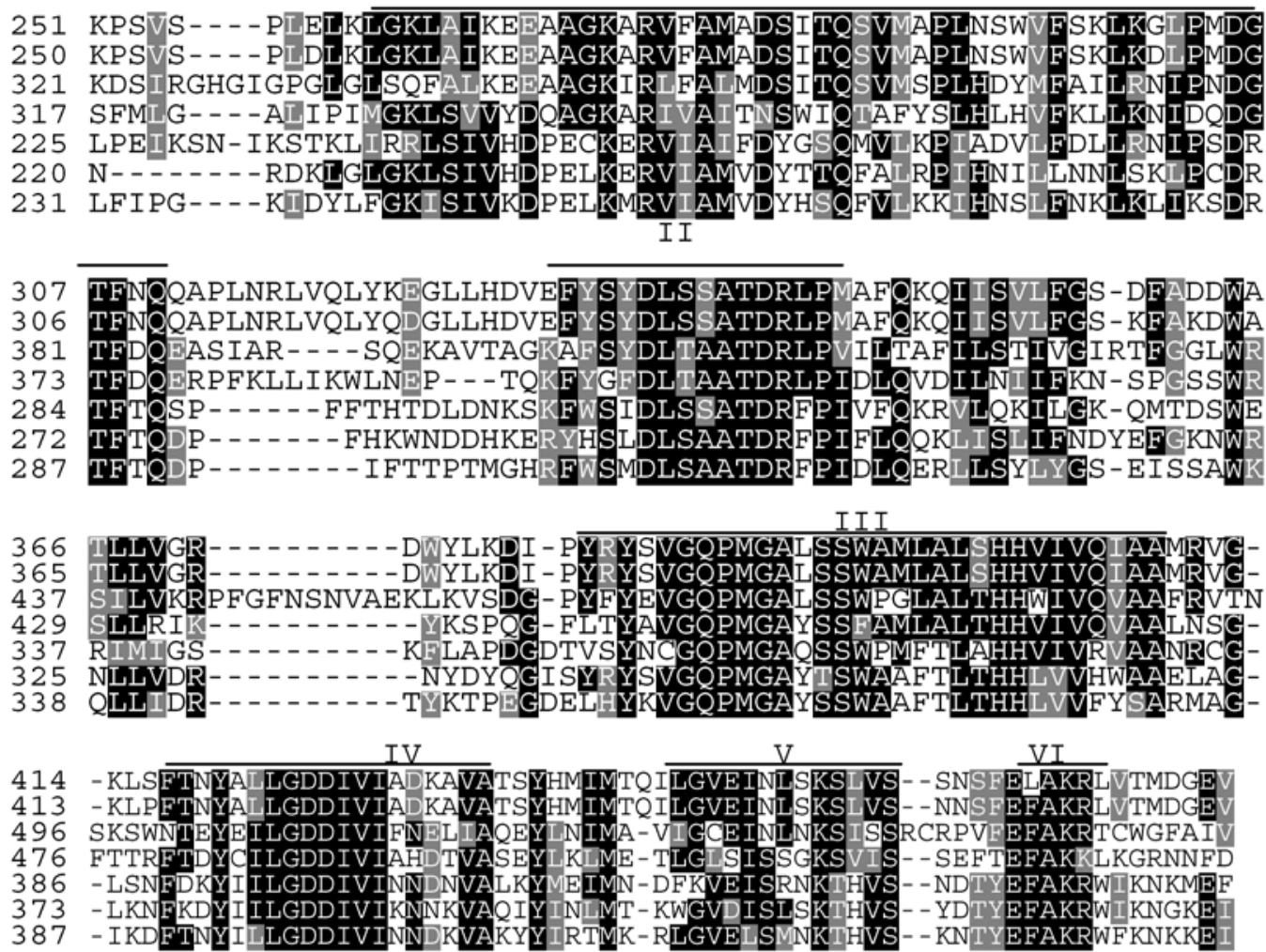

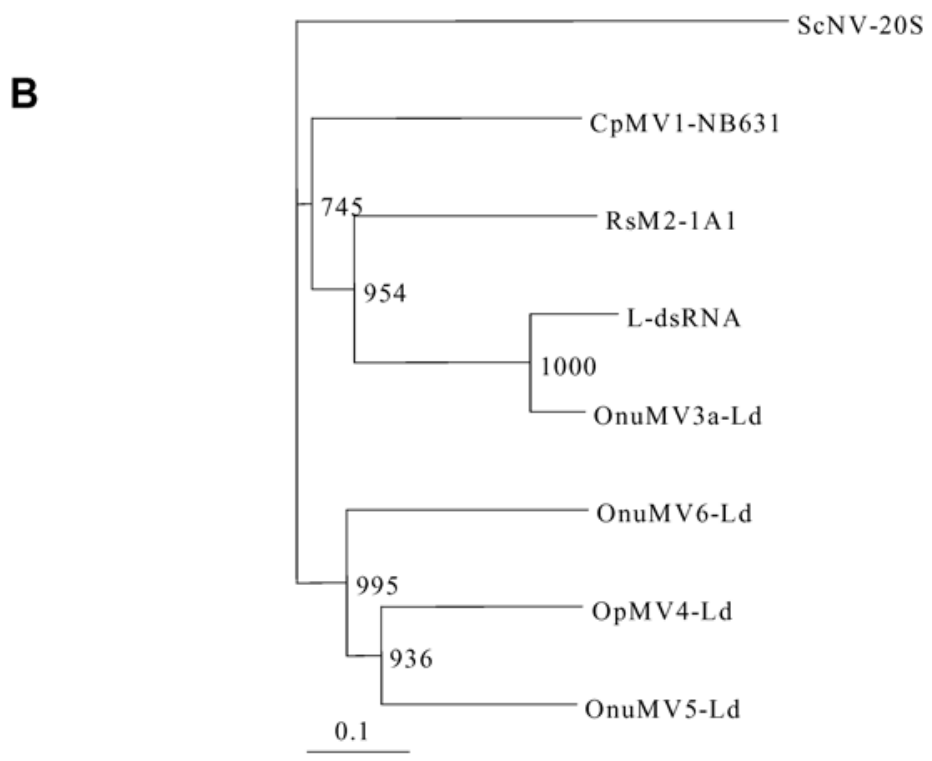

Fig. 2. A, Alignments and B, phylogram of the conserved amino acid sequence motifs in the RNA-dependent RNA polymerase (RdRp)-like proteins encoded by mitochondrial virus and related double-stranded (ds)RNAs. Virus notations and GeneBank accession numbers: Sclerotinia homoeocarpa large dsRNA (LdsRNA, AY172454), Cryphonectria parasitica NB631 mitochondrial dsRNA (CpMV1-NB631, L31849), Rhizoctonia solani 1A1 M2 dsRNA (RsM2-1A1, U51331). OnuMV3a-Ld, OnuMV4-Ld, OnuMV5-Ld, and OnuMV6-Ld are Ophiostoma novo-ulmi dsRNAs 3a, 4, 5, and 6 (AJ004930, AJ132754, AJ132755, and AJ132756), respectively. Identical amino acid residues are black-shaded and similar amino acid residues are gray-shaded. Alignments were performed with the Clustal W program (42). Phylogram was drawn with the Clustal W program and displayed with the treeview program. Numbers at nodes represent bootstrap values out of 1,000 trials. The Saccharomyces cerevisiae 20S RNA narnavirus (ScNV-20S) was used as an outgroup. 
2484R (5'-CTTTAAGCGTAGTTTCCCCAC-3'), which amplified 450-bp sscDNA fragments corresponding to nucleotides 2,069 to 2,518 of the L-dsRNA. Hybridization was conducted overnight at $42^{\circ} \mathrm{C}$ with $20 \mathrm{ng}$ of probe per milliliter of hybridization buffer. Blots were washed twice in $2 \times$ washing solution $(2 \times$ SSC and $0.1 \%$ SDS) for $15 \mathrm{~min}$ each at room temperature, and then twice in $0.5 \times$ washing solution $(0.5 \times \mathrm{SSC}$ and $0.1 \% \mathrm{SDS})$ at $68^{\circ} \mathrm{C}$ for $15 \mathrm{~min}$ each. An immunological method was used for detection, according to the manufacturer's instructions (Boehringer Mannheim).

\section{RESULTS}

Nucleotide sequence of the L-dsRNA. Sequence analysis showed that the L-dsRNA of $S$. homoeocarpa has 2,632 nucleotides, and is relatively enriched in A and $\mathrm{U}(61.0 \%)$. The A- and U-rich character of the L-dsRNA is similar to that of fungal and plant mitochondrial genomes $(13,33,43)$ and several previously characterized fungal mitochondrial dsRNA viruses $(21,22,35)$. The nucleotide sequence of the L-dsRNA is available from the GeneBank database with accession number AY172454.

Open reading frame and codon usage analysis. The nucleotide sequence of the L-dsRNA was examined for the presence of open reading frames (ORFs) on both strands. Both cytoplasmic and mitochondrial locations of dsRNA have been reported in filamentous fungi (20-22,35,40); therefore, cytoplasmic and mitochondrial codon usages were used in the analysis. When normal cytoplasmic codon usage was invoked, no long ORFs were found on either strand. When using mitochondrial codons, a large ORF, termed ORF I, with the potential to encode 720 amino acids (aa) was found to extend almost the full length of the + strand RNA in frame 2 (Fig. 1B). This ORF contains 12 UGA codons and terminates in a UAA codon. Mitochondria in most ascomycete fungi use UGA to encode tryptophan rather than as a translation terminator and UAA is the preferred, or sometimes the only, stop codon used in fungal mitochondria (33). The codon usage of this ORF has a preference of $\mathrm{A}$ or $\mathrm{U}$ over $\mathrm{G}$ or $\mathrm{C}$ at the third or wobble position, and $33.3,33.6,17.2$, and $15.8 \%$ of the total codons had $\mathrm{A}, \mathrm{U}, \mathrm{G}$, and $\mathrm{C}$ at this position, respectively. This trend is in accordance with codon usage in the mitochondria of higher fungi $(13,33,43)$.

On the complementary, - strand of the L-dsRNA, a small ORF, designated ORF II, with the potential to encode a protein of 182 aa, was detected (Fig. 1B). This ORF contained two UGA codons but a Blast search (1) did not find any homologous amino acid sequences.

High sequence similarity between L-dsRNA and $O$. novoulmi mitovirus 3a from $O$. novo-ulmi. Analysis of ORF I revealed that it has amino acid sequence motifs typical of RNAdependent RNA polymerases (RdRp) (Fig. 2A). Motifs II to VI are similar to RdRp motifs described by Bruenn $(5,6)$, Habili and Symons (18), Koonin (26), and Poch et al. (34). Motif I is a combination of motifs X and Y described by Hong et al. (21).

Sequence analysis of the L-dsRNA nucleotide and RdRp-like protein also revealed that this dsRNA was related to those of a Cryphonectria parasitica mitovirus 1 (CpMV1-NB631) from $C$. parasitica (35), four proposed mitovirus species (O. novo-ulmi mitovirus 3a-Ld [OnuMV3a-Ld], OnuMV4-Ld, OnuMV5-Ld, and OnuMV6-Ld) from O. novo-ulmi $(21,22)$, and a mitovirus-like dsRNA (M2 dsRNA) from $R$. solani (27). Figure 2A presents a partial alignment to show the conserved motifs of amino acid sequences among the RdRp-like proteins encoded by these mitoviruses and mitovirus-like dsRNAs. Phylogenic analysis indicated that the L-dsRNA and OnuMV3a-Ld were more closely related to each other than to other mitovirus and mitovirus-like dsRNA (Fig. 2B).

Nucleotide sequence identities between pairwise combinations of these dsRNA viruses are shown in Table 1. Pairwise comparisons detected the highest nucleotide sequence identity $(92.4 \%)$ between the L-dsRNA and mitovirus OnuMV3a-Ld from $O$. novo$u l m i$, the causal agent of Dutch elm disease. In contrast, sequence identities among other dsRNA viruses ranged from 39.4 to $53.3 \%$. Amino acid sequence comparisons among putative RdRp-like proteins and the conserved motifs of RdRps also revealed high similarity between the L-dsRNA of $S$. homoeocarpa and the OnuMV3a-Ld dsRNA of O. novo-ulmi $(95.1$ and $98.6 \%$, respectively). There were only 35 mismatches of amino acid residues between the two complete putative RdRp-like proteins and, among

TABLE 1. Nucleotide sequence identities of mitochondrial viruses and related double-stranded RNAs ${ }^{\mathrm{a}}$

\begin{tabular}{|c|c|c|c|c|c|c|}
\hline & OnuMV3a-Ld & OnuMV4-Ld & OnuMV5-Ld & OnuMV6-Ld & CpMV1-NB631 & RsM2-1A1 \\
\hline L-dsRNA & 92.4 & 46.3 & 44.9 & 44.8 & 44.1 & 43.1 \\
\hline OnuMV3a-Ld & $\ldots$ & 48.5 & 45.5 & 45.7 & 45.2 & 41.6 \\
\hline OnuMV4-Ld & $\ldots$ & $\ldots$ & 53.3 & 52.3 & 47.7 & 42.8 \\
\hline OnuMV5-Ld & $\begin{array}{l}\cdots \\
\ldots\end{array}$ & $\begin{array}{l}\cdots \\
\ldots\end{array}$ & $\ldots$ & 52.3 & 47.1 & 39.4 \\
\hline OnuMV6-Ld & $\ldots$ & $\ldots$ & $\ldots$ & $\ldots$ & 45.4 & 39.8 \\
\hline CpMV1-NB631 & $\ldots$ & $\ldots$ & $\begin{array}{l}\cdots \\
\ldots\end{array}$ & $\begin{array}{l}\cdots \\
\ldots\end{array}$ & $\ldots$ & 42.6 \\
\hline
\end{tabular}

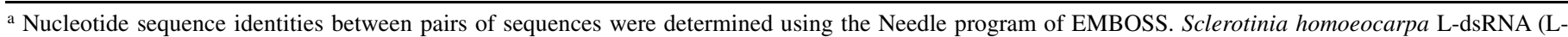
dsRNA, present study); Ophiostoma novo-ulmi Ld mitochondrial viruses: OnuMV3a-Ld (RNA-3a), OnuMV4-Ld (RNA-4), OnuMV5-Ld (RNA-5), OnuMV6Ld (RNA-6) (21,22); Cryphonectria parasitica NB631 mitochondrial dsRNA (CpMV1-NB631) (35); and $R$. solani 1A1 M2 dsRNA (RsM2-1A1) (27).

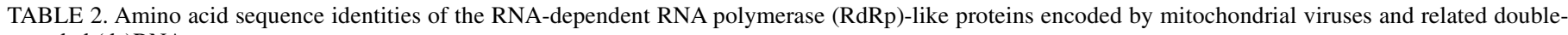
stranded (ds)RNAs ${ }^{\mathrm{a}}$

\begin{tabular}{|c|c|c|c|c|c|c|c|}
\hline & L-dsRNA & OnuMV3a-Ld & OnuMV4-Ld & OnuMV5-Ld & OnuMV6-Ld & CpMV1-NB631 & RsM2-1A1 \\
\hline L-dsRNA & $\ldots$ & 95.1 & 22.2 & 21.6 & 24.4 & 23.5 & 26.3 \\
\hline OnuMV3a-Ld & 98.6 & $\ldots$ & 21.5 & 21.8 & 24.4 & 24.3 & 25.3 \\
\hline OnuMV4-Ld & 52.9 & 53.6 & $\ldots$ & 31.7 & 28.4 & 20.8 & 17.6 \\
\hline OnuMV5-Ld & 52.9 & 53.6 & 71.7 & $\ldots$ & 33.9 & 21.6 & 20.7 \\
\hline OnuMV6-Ld & 51.4 & 52.2 & 61.6 & 65.2 & $\ldots$ & 23.4 & 22.2 \\
\hline CpMV1-NB631 & 56.5 & 57.2 & 49.3 & 50.0 & 50.0 & $\ldots$ & 21.6 \\
\hline RsM2-1A1 & 63.0 & 63.8 & 47.8 & 45.7 & 50.7 & 55.8 & $\ldots$ \\
\hline
\end{tabular}

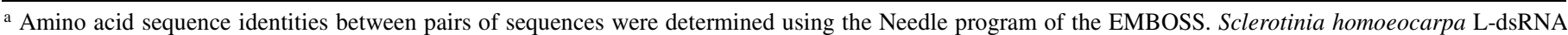
(L-dsRNA, present study); Ophiostoma novo-ulmi Ld mitochondrial viruses: OnuMV3a-Ld (RNA-3a), OnuMV4-Ld (RNA-4), OnuMV5-Ld (RNA-5), OnuMV6-Ld (RNA-6) (21,22); Cryphonectria parasitica NB631 mitochondrial dsRNA (CpMV1-NB631) (35); and $R$. solani 1A1 M2 dsRNA (RsM2-1A1) (27). Top right triangle: amino acid sequence identities between complete RdRp-like protein sequences. Absent amino acids in gaps, including terminal gaps, were treated as mismatches. Bottom left triangle: amino acid sequence identities between conserved RdRp sequence motifs I to VI. There were no gaps in these alignments. 
these 35 mismatches, 24 were chemically similar amino acid residues such as substitutions from isoleucine to valine and glutamic acid to aspartic acid. Sequence comparisons with the other viruses ranged from 17.6 to $33.9 \%$ for the complete RdRp-like proteins and from 45.7 to $71.7 \%$ for the conserved RdRp motifs (Table 2). A Northern blot analysis of the dsRNAs extracted from isolate Sh12B of $S$. homoeocarpa and isolate $\log 1 / 38 \mathrm{~d}^{2}$ of $O$. novo-ulmi is shown in Figure 3A. cDNA probes prepared from the L-dsRNA of $S$. homoeocarpa hybridized with both the L-dsRNA and the OnuMV3a-Ld dsRNA of $O$. novo-ulmi, indicating that these two dsRNAs were closely related.

Detection of the single-stranded (positive stranded) form of the L-dsRNA. The ssRNA ( $2 \mathrm{M} \mathrm{LiCl}$ fraction) and dsRNA (5 M $\mathrm{LiCl}$ fraction) fractions recovered from hypovirulent isolate Sh12B of S. homoeocarpa were electrophoresed in a $1.4 \%$ nondenaturing agarose gel, blotted onto a nylon membrane, and hybridized with strand-specific DNA probes. In the dsRNA fraction, a band was detected by both + strand-specific and - strand-specific probes (Fig. 3B, lanes 1 and 3), confirming that it is double stranded. In the ssRNA fraction, a band that migrated faster than the dsRNA band was detected only by the + strand-specific probe, confirming its single-stranded nature (Fig. 3B, lanes 2 and 4). Failure to detect the ssRNA using the - strand-specific probe indicates the ssRNA is the positive strand of the L-dsRNA. Therefore, nucleic acid extracts from isolate Sh12B of $S$. homoeocarpa contain both dsRNA and positive-stranded ssRNA encoding the RdRp-like protein. The - strand-specific probe covers the ORF II region on the - strand of the L-dsRNA; failure to detect either full-length or subgenomic - strand ssRNA suggests that this ORF probably is not expressed. Because the amounts of ssRNA and dsRNA analyzed were derived from the same amount of total nucleic acids, and the RNA was denatured and partially fragmented with alkali before blotting, all RNA samples should have been in the ssRNA form prior to transfer and hybridization. Therefore, the relatively strong signal of ssRNA and weak signal of dsRNA from Northern blotting may indicate that isolate Sh12B of S. homoeocarpa contained more single, + stranded RNA than dsRNA, which is similar to the previous report for OnuMv3a-Ld dsRNA in $O$. novo-ulmi (21).

Comparison of terminal sequences and potential secondary structures between L-dsRNA and OnuMV3a dsRNA. The LdsRNA in hypovirulent isolate Sh12B of S. homoeocarpa is 2,632 bp long, whereas the dsRNA genome of $O$. novo-ulmi mitovirus 3a-Ld is 2,617 bp long. One major difference between these two dsRNAs is that the L-dsRNA of $S$. homoeocarpa has four GC pairs at the $5^{\prime}$ terminus and six CG pairs at the $3^{\prime}$ terminus. The $O$. novo-ulmi mitovirus 3a-Ld dsRNA lacks this characteristic (Fig. $4 \mathrm{~A}$ ). The previously reported $R$. solani M2 dsRNA also has terminal GC pairs (27). In addition, the nucleotides of the two dsRNAs are highly conserved at the $5^{\prime}$ terminus, but the nucleotide sequences at the $3^{\prime}$ terminus have more variations. The 5'- and $3^{\prime}$ untranslated sequences of the + strand of the L-dsRNA were examined for potential secondary structures using the program MFOLD (28), because these structures may act as promoters for RNA replication (7). It was shown that the $5^{\prime}$ terminal nucleotides (nucleotides 4 to 25) can be folded into a stem-loop structure, and the $3^{\prime}$ terminal sequence (nucleotides 2,511 to 2,628) also could be folded into a potentially stable, although imperfect, stem-loop structure (Fig. 4B). Similarly, the 5' terminal 1 to $20 \mathrm{nt}$ and $3^{\prime}$ terminal 2,502 to 2,617 nt of OnuMV3a-Ld also could be folded into stem-loop structures as reported by Hong et al. (21). The predicted $5^{\prime}$ terminal stem-loop structures of the two RNAs are quite similar (one GC pair difference on the stem structure), whereas the $3^{\prime}$ terminal stem-loop structures differed, to some extent, on both the loop and the stem structures. Both the + strands of the L-dsRNA and OnuMV3a-Ld dsRNA do not have inverted complementarity with the potential to form panhandle structures as reported for OnuMV4-Ld and OnuMV6-Ld (22).

\section{DISCUSSION}

This is the first report that a hypovirulence-associated, dsRNA virus naturally occurs in two taxonomically distinct fungi. Owing to their intracellular mode of transmission, which is known to oc-
A

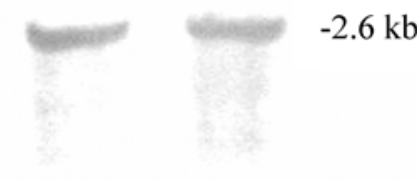

B 1

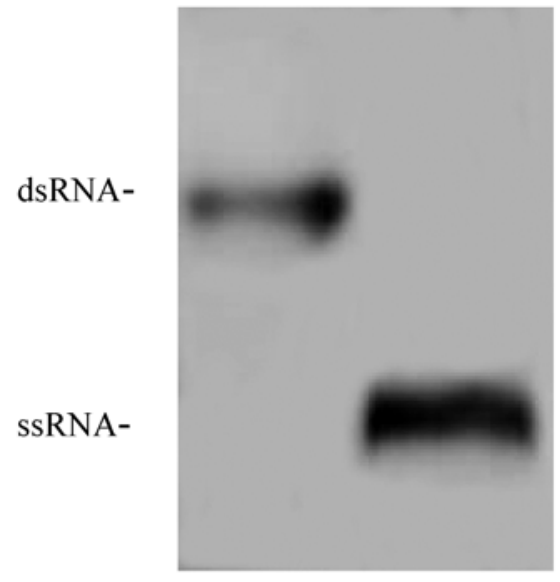

(+) strandspecific probe

2

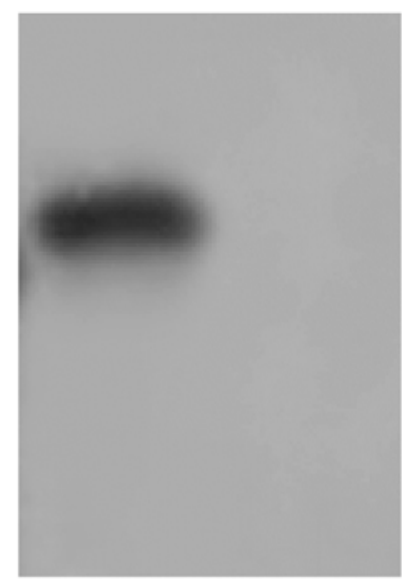

(-) strandspecific probe

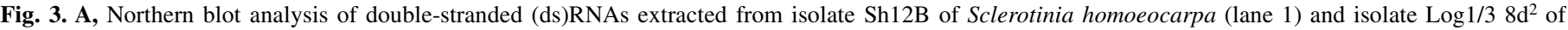

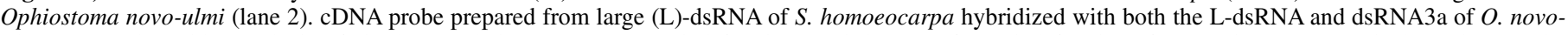

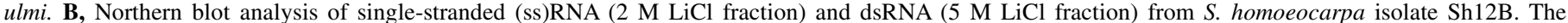

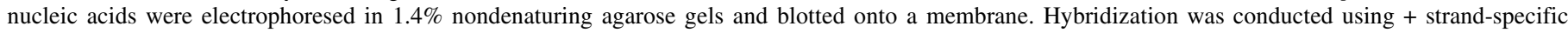

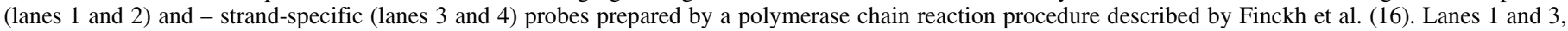
dsRNA samples. Lanes 2 and 4, ssRNA samples. 
cur only through hyphal anastomosis and does not involve external infection, the natural host ranges of these dsRNA viruses have been considered to be limited to individuals within the same or closely related vegetative compatibility groups (2). The characterized hypoviruses have not been reported to naturally infect other fungal species outside of $C$. parasitica. Similarly, the nucleotide sequences of previously described mitovirus- and mitovirus-like dsRNAs in different fungi, even in the same isolate of a fungus, were poorly conserved. The nucleotide sequence identities among these mitoviruses ranged from 39.4 to $53.3 \%$ (Table 1), and the amino acid sequence identities of the RdRp-like proteins encoded by these mitoviruses ranged from 17.6 to $33.9 \%$ (Table 2). These dsRNA viruses have been proposed to constitute separate virus species $(21,22)$.

A virus species has been defined as a polythetic class of viruses that constitute a replicating lineage and occupy a particular eco- logical niche $(44,45)$. Thus, a virus species is not a physical entity but a collection of virus strains or isolates with closely similar properties. The RdRps lack proofreading ability; therefore, a relatively high mutation frequency occurs during replication of an RNA virus genome, and viral RNA molecules may consist of populations of closely related molecules or "quasi-species" (15). The accepted genomic sequence for a virus represents the predominant sequence, or a consensus sequence, derived from characterization of a number of cDNA clones isolated from the population of sequences present in virally infected cells. In virus taxonomy, genotype should be the ultimate criterion on which to assign viruses to their groups and genome sequence relatedness has been used in species demarcation (47). Although no particular degree of genome sequence dissimilarity can be used as a cutoff point to differentiate between two virus species, generally, less than $50 \%$ sequence identity at the protein level reflects a species

A

\section{5'-terminus}

L-dsRNA

OnuMV3a-Ld

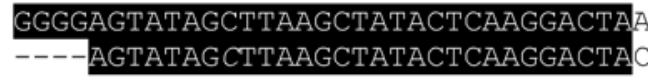

GTCCTCCCTCAGAGAATTA-CACCCCTCTGAGATGG

\section{3'-terminus}

$\begin{array}{lrl}\text { L-dsRnA } & 2525 & \text { TTGGCTTTCAAATATGACTACTCCTTTTAATACATGGGCCCGATAAACCAGGGG } \\ \text { OnumV3a-Ld } 2515 \text { TTGTCTTTCAAATATGACTACTCCCTCTCATCCATTGGTCTGTAAACCAATGT }\end{array}$

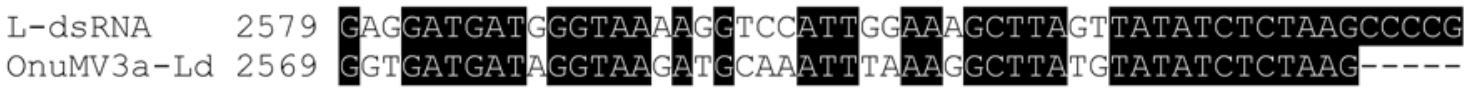

OnuMV3a-Ld 2569 GGTGATGATAGGTAAGATGCAAATTTAAAGGCTTATGTATATCTCTAAG-----

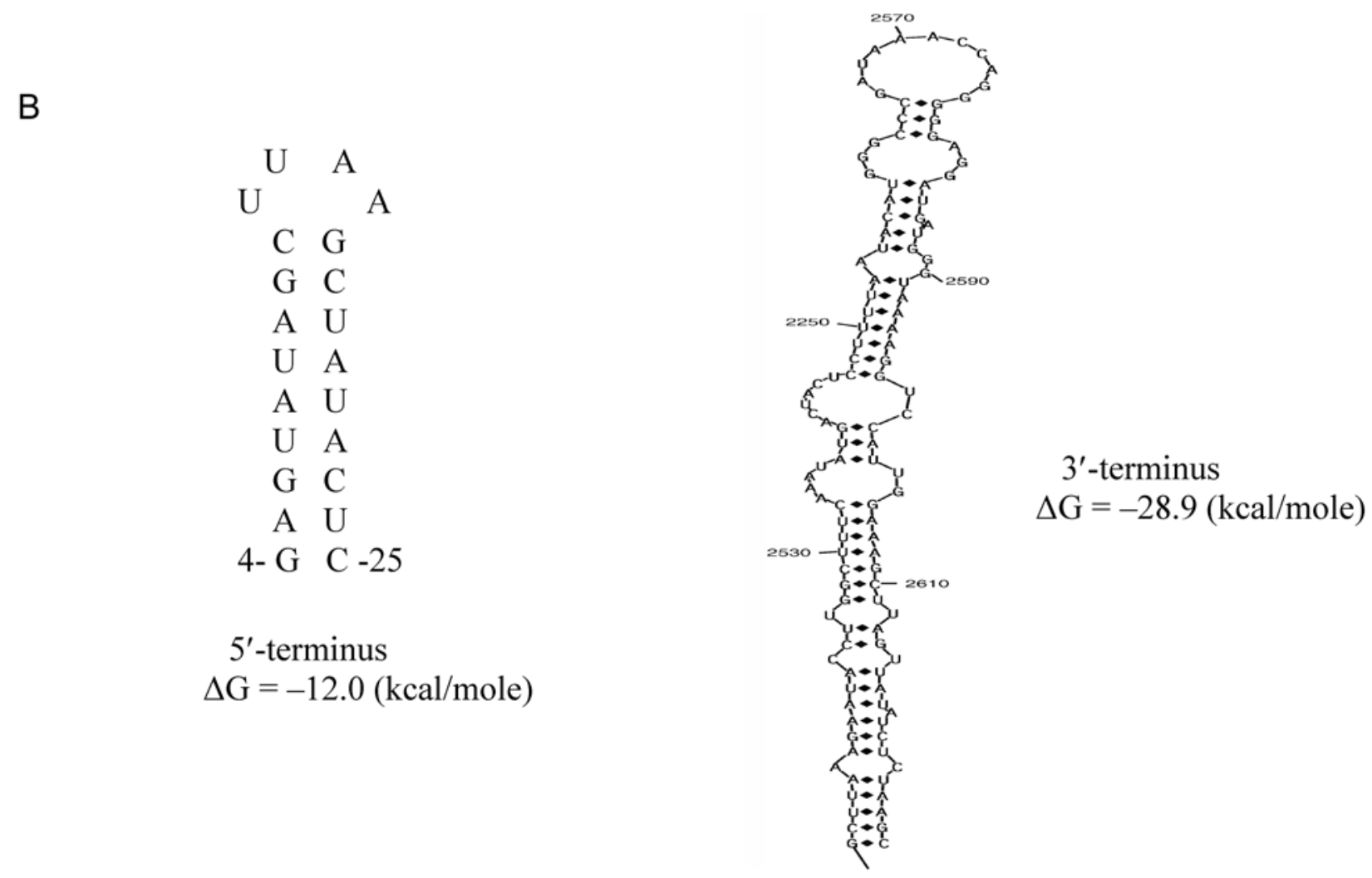

Fig. 4. A, Comparison of $5^{\prime}$ and $3^{\prime}$ terminal sequences of the + strands between the large double-stranded (L-ds)RNA in isolate Sh12B of Sclerotinia homoeocarpa and OnuMV3a-Ld dsRNA in Ophiostoma novo-ulmi. Identical nucleotides are shaded. The L-dsRNA has four GC pairs at the 5' terminus and six CG pairs at the $3^{\prime}$ terminus, while the OnuMV3a-Ld dsRNA lacks these characteristics. B, Potential secondary structures of the $5^{\prime}$ terminal 4 to 25 nucleotides and $3^{\prime}$ terminal nucleotides 2,511 to 2,528 of L-dsRNA (positive strand). The terminal sequences were folded and the free energy calculated with the MFOLD program (28). The 5' and 3' terminal stem-loop structures folded by OnuMV3a-Ld RNA have been described by Hong et al. (21). 
difference. A good example is the family Potyviridae, in which genome and coat protein sequence comparisons established a clear-cut distinction between each of the different taxonomic levels: strains, species, and genera (39). The sequence identity between distinct species ranged from 38 to $71 \%$ (average $54 \%$ ), while that between strains of one virus species ranged from 90 to $99 \%$ (average 95\%) (47). In hypoviruses, the overall nucleotide sequence identity was $38 \%$ between CHV1-EP713 and CHV3-GH2 as well as between CHV2-NB58 and CHV3-GH2, and the overall sequence identity between CHV1-EP713 and CHV2-NB58 was $\approx 60 \%(20,40)$. In comparison, the nucleotide and amino acid sequence identities between the two characterized strains of CHV1, in isolates EP713 and Euro7, were 87 to 93 and 90 to $98 \%$, respectively (10).

In the present study, the hypovirulence-associated dsRNA (LdsRNA) from $S$. homoeocarpa was characterized as a mitovirus. Mitoviruses represent the simplest of all known autonomously replicating viruses because they have no capsid and consist of only a single naked RNA genome that encodes only an RdRp-like protein that is required to replicate the RNA. The fact that this virus shares $92.4 \%$ nucleotide and $95.1 \%$ amino acid sequence identities with $O$. novo-ulmi mitovirus 3a (OnuMV3a-Ld), a mitovirus from $O$. novo-ulmi, suggests that these dsRNAs should be considered as conspecific (e.g., two strains of the same virus species, OnuMV3a). Because strains of a mitovirus can be found in more than one fungal species, this strain should be assigned the name O. novo-ulmi mitovirus 3a-Sh12B (OnuMV3a-Sh12B; Sh12B indicates isolate Sh12B of $S$. homoeocarpa, where the viral strain was derived), based on the nomenclature of the International Committee on Taxonomy of Viruses (48).

The pathogen that causes dollar spot of turf grass currently is classified as $S$. homoeocarpa, whereas Dutch elm disease is caused by $O$. ulmi and $O$. novo-ulmi. Although the taxonomic status of $S$. homoeocarpa remains controversial and several proposals have been put forward to reclassify this fungus $(25,36)$, it is obvious that these two fungi are not phylogenically closely related. S. homoeocarpa produces apothecia and is classed as a Discomycete, whereas $O$. novo-ulmi produces perithecia and is classed as a Pyrenomycete. The high sequence identities at both the nucleotide (92.4\%) and amino acid levels (95.1\%) do not support the hypothesis that the virus infection could have arisen early in their phylogeny before the two fungal species diverged. A long period of coevolution with their fungal hosts would have resulted in a significant sequence divergence of their dsRNA genomes, because RNA mycoviruses are believed to evolve at a more rapid rate than their host DNA genomes (8). The natural occurrence of this dsRNA virus in these two fungi strongly suggests that horizontal transmission of this virus may have occurred between $S$. homoeocarpa and $O$. nomo-ulmi or $O$. ulmi. For the same reason, the high sequence identities also suggest that horizontal transmission of this virus either occurred commonly or recently between the two fungi.

Transmission of genetic materials between unrelated fungi has been reported and these fungi usually inhabit the same ecological niche. Brasier et al. (4) identified rare interspecific hybrids of $O$. $u l m i$ and $O$. novo-ulmi in natural populations of these fungi where their distributions overlapped, and hyphal fusion may serve as genetic bridges facilitating the transfer of genetic materials such as dsRNAs between these two species. Kempken (24) demonstrated in vitro transmission of a mitochondrial plasmid from Ascobolus immerses to Podospora anserine. Kellner et al. (23) reported in vitro transfer of genetic material from the mycoparasite Parasitella parasitica to its host Absidia glanca. Recently, Melzer et al. (29) demonstrated transmission of a dsRNA from S. sclerotiorum to $S$. minor through hyphal anastomosis. Hybrids of the fungal species were not detected but transitory hyphae fusions appeared to be sufficient to enable dsRNA to be transmitted between these Sclerotinia spp. There are no reports that $O$. novo-ulmi and $S$. homoeocarpa share any common hosts, but their hosts can in- habit the same ecological niche. Therefore, there are potential ecological interactions between these two fungi. Transmission of dsRNA viruses may occur from transient fusions between hyphae of these two fungi, and mycophagous animals such as mites and nematodes also may transmit dsRNA viruses.

Although OnuMV3a naturally occurs in S. homoeocarpa and $O$. novo-ulmi, it only induces hypovirulence in $S$. homoeocarpa. Multiple mitoviruses were present in the diseased isolate $\log 1 / 3$ $8 \mathrm{~d}^{2}$ of O. novo-ulmi, but analysis of dsRNA segments in singleconidial isolates derived from this isolate, which lost various combinations of dsRNA segments, indicated that OnuMV3a-Ld was not the cause of hypovirulence, and OnuMV4-Ld may be responsible for hypovirulence (11). Similarly, isolates of Cryphonectria radicalis, C. havanensis, C. cubensis, and Endothia gyrosa were artificially infected by synthetic transcripts of the hypovirus CHV1-EP713, from C. parasitica, and this hypovirus also had different phenotypic effects on these fungal species (9). Therefore, it seems that a mitovirus may have differential effects on its fungal hosts.

Little information is available on the host range of hypovirulence-associated dsRNAs because they are only transmitted cytoplasmically through hyphal anastomoses, and there are no techniques to transmit most dsRNA viruses by simple infection or transduction. However, fungi have been artificially infected by hypovirulence-associated dsRNAs from other species. In addition to the closely related species discussed above, strains of Valsa ceratosperma and Phomopsis G-type, which are different genera of the order Diaporthales, also were artificially infected by the synthetic cDNA transcript of hypovirus CHV1-EP713. These infections resulted in hypovirulence in these fungal species, further extending the host range of the hypovirus (38). In another report, a dsRNA from $S$. sclerotiorum was transmitted to $S$. minor through hyphal anastomosis and this dsRNA also induced hypovirulence in its new host (29). The results of the present study suggest that the hypovirulence-associated dsRNA virus OnuMV3a can infect distinct fungi in nature. All these results indicate that hypovirulence-associated dsRNA viruses can have a host range and that it is possible to extend the utility of these viruses in biological control of plant diseases.

\section{ACKNOWLEDGMENTS}

This research was financially supported by the Natural Science and Engineering Research Council of Canada (NSERC) and the Ontario Ministry of Agriculture and Food (OMAF). We thank C. M. Brasier of the Forest Research Station, Alice Holt Lodge, Farnham, Surrey, UK, for providing isolates of Ophiostoma novo-ulmi; and M. S. Melzer for technical assistance.

\section{LITERATURE CITED}

1. Altschul, S. F., Gish, W., Miller, W., Myers, E. W., and Lipman, D. J. 1990. Basic local alignment search tool. J. Mol. Biol. 5:403-410.

2. Anagnostakis, S. L. 1982. Biological control of chestnut blight. Science 215:466-471.

3. Baltimore, D. 1966. Purification and properties of poliovirus doublestranded RNA. J. Mol. Biol. 18:421-428.

4. Brasier, C. M., Kirk, S. A., Pipe, N. D., and Buck, K. W. 1998. Rare interspecific hybrids in natural populations of the Dutch elm disease pathogens Ophiostoma ulmi and O. novo-ulmi. Mycol. Res. 102: 45-57.

5. Bruenn, J. A. 1991. Relationships among the positive strand and doublestrand RNA viruses as viewed through their RNA-dependent RNA polymerases. Nucleic Acids Res. 19:217-226.

6. Bruenn, J. A. 1993. A closely related group of RNA-dependent RNA polymerases from double-stranded RNA viruses. Nucleic Acids Res. 21:5667-5669.

7. Buck, K. W. 1996. Comparison of the replication of positive-stranded RNA viruses of plants and animals. Adv. Virus Res. 47:159-251.

8. Buck, K. W. 1998. Molecular variability of viruses of fungi. Pages 53-72 in: Molecular Variability of Fungal Pathogens. P. D. Bridge, Y. Couteaudier, and J. M. Clarkson, eds. CAB International, Wallingford, UK. 
9. Chen, B., Chen, C. H., Bowman, B. H., and Nuss, D. L. 1996. Phenotypic changes associated with wild-type and mutant hypovirus RNA transfection of plant pathogenic fungi phylogenetically related to Cryphonectria parasitica. Phytopathology 86:301-310.

10. Chen, B., and Nuss, D. L. 1999. Infectious cDNA clone of hypovirus CHV1-Euro7: A comparative virology approach to investigate virusmediated hypovirulence of the chestnut blight fungus Cryphonectria parasitica. J. Virol. 73:985-992.

11. Cole, T. E., Müller, B., Hong, Y., Brasier, C. M., and Buck, K. W. 1998. Complexity of virus-like double-stranded RNA elements in a diseased isolate of the Dutch elm disease fungus, Ophiostoma novo-ulmi. J. Phytopathol. 146:593-598.

12. Craven, M. G., Pawlyk, D. M., Choi, G. H., and Nuss, D. L. 1993. Papain-like protease p29 as symptom determinant encoded by a hypovirulence-associated virus of the chestnut blight fungus. J. Virol. 67:6513-6521.

13. Cummings, D. J., McNally, K. L. Domenico, J. M., and Matsuura, E. T. 1990. The complete DNA sequence of the mitochondrial genome of Podospora anserina. Curr. Genet. 17:375-402.

14. Diaz-Ruiz, J. R., and Kaper, J. M. 1978. Isolation of viral double-stranded RNA using a $\mathrm{LiCl}$ fractionation procedure. Prep. Biochem. 8:1-13.

15. Eigen, M., and Biebriecher, C. 1988. Sequence space and quasispecies distribution. Pages 211-245 in: RNA Genetics. E. Domingo, J. J. Holland, and P. Ahlquist, eds. CRC Press, Boca Raton, FL.

16. Finckh, U., Lingenfelter, P. A., and Myerson, D. 1991. Producing singlestranded DNA probes with the Taq DNA polymerase: A high yield protocol. BioTechniques 10:35-38.

17. Frohman, M. A., Dush, M. K., and Martin, G. R. 1988. Rapid production of full-length cDNAs from rare transcripts: Amplification using a single gene-specific oligonucleotide primer. Proc. Natl. Acad. Sci. USA 85:8998-9002.

18. Habili, N., and Symons, R. H. 1989. Evolutionary relationship between luteoviruses and other RNA plant viruses based on sequence motifs in their putative RNA polymerases and nucleic acid helicases. Nucleic Acids Res. 17:9543-9555.

19. Hillman, B. I., Fulbright, D. W., Nuss, D. L., and Van Alfen, N. K. 2000. Family Hypoviridae. Pages 515-520 in: Virus Taxonomy: Seventh Report of the International Committee on Taxonomy of Viruses. M. H. V. Van Regenmortel, C. M. Fauquet, D. H. L. Bishop, E. B. Carstens, M. K. Estes, S. M. Lemon, J. Maniloff, M. A. Mayo, D. J. McGeoch, C. R. Pringle, and R. B. Wichner, eds. Academic Press, San Diego, CA.

20. Hillman, B. I., Halpern, B. T., and Brown, M. P. 1994. A viral dsRNA element of the chestnut blight fungus with a distinct genetic organization. Virology 201:241-250.

21. Hong, Y., Cole, T. E., Brasier, C. M., and Buck, K. W. 1998. Evolutionary relationships among putative RNA-dependent RNA polymerases encoded by a mitochondrial virus-like RNA in the Dutch elm disease fungus, Ophiostoma novo-ulmi, by other viruses and virus-like RNAs and by the Arabidopsis mitochondrial genome. Virology 246:158-169.

22. Hong, Y., Dover, S. L., Cole, T. E., Brasier, C. M., and Buck, K. W. 1999. Multiple mitochondrial viruses in an isolate of the Dutch elm disease fungus Ophiostoma novo-ulmi. Virology 258:118-127.

23. Kellner, M., Burmester, A., Wöstemeyer, A., and Wöstemeyer, J. 1993. Transfer of genetic information from the mycoparasite Parasitella parasitica to its host Absidia glauca. Curr. Genet. 23:334-337.

24. Kempken, F. 1995. Horizontal transfer of a mitochondrial plasmid. Mol. Gen. Genet. 248:89-94.

25. Kohn, L. M. 1979. A monographic revision of the genus Sclerotinia. Mycotaxon 9:365-444.

26. Koonin, E. V. 1991. The phylogeny of RNA-dependent RNA polymerases of positive-strand RNA viruses. J. Gen. Virol. 72:2197-2206.

27. Lakshman, D. K., Jian, J., and Tavantzis, S. M. 1998. A double-stranded RNA element from a hypovirulent strain of Rhizoctonia solani occurs in DNA form and is genetically related to the pentafunctional AROM protein of the shikimate pathway. Proc. Natl. Acad. Sci. USA 95:6425-6429.

28. Mathews, D. H., Sabina, J., Zuker, M., and Turner, D. H. 1999. Expanded sequence dependence of thermodynamic parameters improves prediction of RNA secondary structure. J. Mol. Biol. 288:911-940.

29. Melzer, M. S., Ikeda, S. S., and Boland, G. J. 2002. Interspecific transmission of double-stranded RNA and hypovirulence from Sclerotinia sclerotiorum to $S$. minor. Phytopathology 92:780-784.
30. Nuss, D. L. 1992. Biological control of chestnut blight: An example of virus mediated attenuation of fungal pathogenesis. Microbiol. Rev. 56:561-576.

31. Nuss, D. L. 1996. Using hypoviruses to probe and perturb signal transduction processes underlying fungal pathogenesis. Plant Cell 8:18451853.

32. Nuss, D. L., and Koltin, Y. 1990. Significance of dsRNA genetic elements in plant pathogenic fungi. Annu. Rev. Phytopathol. 28:37-58.

33. Paquin, B., Laforest, M. J., Forget, L., Roewer, I., Wang, Z., Longcore, J., and Lang, B. F. 1997. The fungal mitochondrial genome project: Evolution of fungal mitochondrial genomes and their gene expression. Curr. Genet. 31:380-395.

34. Poch, O., Sauvaget, I., Delarue, M., and Tordo, N. 1989. Identification of four conserved motifs among the RNA-dependent RNA polymerase encoding elements. EMBO J. 8:3867-3874.

35. Polashock, J. J., and Hillman, B. I. 1994. A small mitochondrial doublestranded (ds) RNA element associated with hypovirulent strain of the chestnut blight fungus and ancestrally related to yeast cytoplasmic T and W RNAs. Proc. Natl. Acad. Sci. USA 91:8680-8684.

36. Powell, J. F., and Vargas, J. M., Jr. 1997. Mycelial compatibility and systematics of Sclerotinia homoeocarpa. (Abstr.) Phytopathology 87(suppl.):S79.

37. Sanger, F., Nicklen, S., and Coulson, A. R. 1977. DNA sequencing with chain-termination inhibitors. Proc. Natl. Acad. Sci. USA 74:64635467.

38. Sasaki, A., Onoue, M., Kanematsu, S., Suzaki, K., Miyanishi, M., Suzuki, N., Nuss, D. L., and Yoshida, K. 2002. Extending chestnut blight hypovirus host range within Diaporthales by biolistic delivery of viral cDNA. Mol. Plant-Microbe Interact. 15:780-789.

39. Shukla, D., Ward, C. W., and Brunt, A. A. 1994. The Potyviridae. CBA International, Wallingford, UK.

40. Smart, C. D., Yuan, W., Foglia, R., Nuss, D. L., Fulbright, D. L., and Hillman, B. I. 2000. Cryphonectria hypovirus 3, a virus species in the family Hypoviridae with a single open reading frame. Virology 265:6673.

41. Suzuki, N., and Nuss, D. L. 2002. Contribution of protein p40 to hypovirus-mediated modulation of fungal host phenotype and viral RNA accumulation. J. Virol. 76:7747-7759.

42. Thompson, J. D., Higgins, D. G., and Gibson, T. J. 1994. Clustal W. Improving the sensitivity of progressive multiple sequence alignments through sequence weighting, position-specific gap penalties and weight matrix choice. Nucleic Acids Res. 22:4697-4680.

43. Unseld, M., Marienfeld, J. R., Brandt, P., and Brennicke, A. 1997. The mitochondrial genome of Arabidopsis thaliana contains 57 genes in 366,924 nucleotides. Nat. Genet. 15:57-61.

44. Van Regenmortel, M. H. V. 1990. Virus species, a much overlooked but essential concept in virus classification. Intervirology 31:241-254.

45. Van Regenmortel, M. H. V. 2000. Introduction to the species concept in virus taxonomy. Pages 3-16 in: Virus Taxonomy: Seventh Report of the International Committee on Taxonomy of Viruses. M. H. V. Van Regenmortel, C. M. Fauquet, D. H. L. Bishop, E. B. Carstens, M. K. Estes, S. M. Lemon, J. Maniloff, M. A. Mayo, D. J. McGeoch, C. R. Pringle, and R. B. Wickner, eds. Academic Press, San Diego, CA.

46. Walsh, B., Ikeda, S. S., and Boland, G. J. 1999. Biology and management of dollar spot (Sclerotinia homoeocarpa); an important disease of turfgrass. HortScience 34:13-21.

47. Ward, C. W., McKern, N. M., Frenkel, J. J., and Shukla, D. D. 1992. Sequence data as the major criterion for potyvirus classification. Pages 283-297 in: Potyvirus Taxonomy. O. W. Barnett, ed. Springer-Verlag, New York.

48. Wickner, R. B., Esteban, R., and Hillman, B. I. 2000. Family Narnaviridae. Pages 651-656 in: Virus Taxonomy: Seventh Report of the International Committee on Taxonomy of Viruses. M. H. V. Van Regenmortel, C. M. Fauquet, D. H. L. Bishop, E. B. Carstens, M. K. Estes, S. M. Lemon, J. Maniloff, M. A. Mayo, D. J. McGeoch, C. R. Pringle, and R. B. Wickner, eds. Academic Press, San Diego, CA.

49. Zhou, T., and Boland, G. J. 1997. Hypovirulence and double-stranded RNA in Sclerotinia homoeocarpa. Phytopathology 87:147-153.

50. Zhou, T., and Boland, G. J. 1998. Suppression of dollar spot by hypovirulent isolates of Sclerotinia homoeocarpa. Phytopathology 88: 788-794. 